\section{Mécanisme d'action des oncogènes à homéodomaine TLX dans les LAL-T}

Vahid Asnafi ${ }^{1,2,3}$, Pierre Ferrier ${ }^{4,5,6}$

${ }^{1}$ Université de médecine, Paris Descartes Sorbonne Cité,

Paris, France ;

${ }^{2}$ Centre national de la recherche scientifique (CNRS) UMR8147 ;

${ }^{3}$ Département d'hématologie, assistance publique-hôpitaux de Paris (AP-HP), hôpital Necker-Enfants malades, 149, rue de Sèvres, 75015 Paris, France ;

${ }^{4}$ Centre d'immunologie de Marseille-Luminy (CIML), Université Aix-Marseille, UM2, Marseille, France ;

${ }^{5}$ Inserm U1104, Marseille, France ;

${ }^{6}$ CNRS UMR7280, case 906, Campus de Luminy, 13288 Marseille, France. vahid.asnafi@nck.aphp.fr ferrier@ciml.univ-mrs.fr

> Parmi les hémopathies, les leucémies aiguës lymphoblastiques $\mathrm{T}$ ( $L A L-T$ ) représentent 15 à $25 \%$ de l'ensemble des $L A L$ et se caractérisent par un phénotype d'arrêt de maturation à un stade précoce de la différenciation lymphoïde T. Ces affections représentent ainsi un groupe hétérogène de leucémies aiguës dont le trait commun est un blocage de la différenciation cellulaire à un stade donné de la maturation thymique $[1,2]$. Leur pronostic, bien que nettement amélioré grâce aux progrès thérapeutiques récents, reste dans l'ensemble relativement médiocre, les taux de survie avoisinant $50 \%$ à 5 ans chez l'adulte et environ 70 à $80 \%$ chez l'enfant [3]. Dans ce contexte, l'identification de biomarqueurs susceptibles d'être utilisés pour la mise au point de thérapies ciblées représente un enjeu particulièrement important.

\section{Réarrangements V(D)J du TCR} et différenciation des thymocytes

L'oncogenèse des LAL-T est multigénique, avec coexistence de plusieurs dérégulations oncogéniques chez le même patient [4]. Parmi les sousgroupes identifiés, figure l'important groupe TLX marqué par la surexpression des oncogènes $T L X I$ ou $T L X 3$. Ces gènes se caractérisent par la présence d'une séquence d'ADN particulière, appelée homeobox, qui code pour un domaine protéique conservé ou homéodomaine, de structure hélicetour-hélice. Cette structure a la propriété de se lier à l'ADN et d'interagir avec des protéines partenaires. Les facteurs de transcription à homéodomaine sont généralement impliqués dans l'organogenèse, la maintenance de l'homéostasie des tissus. Ils participent au contrôle de multiples fonctions cellulaires dont la croissance, la prolifération et l'apoptose [5]. TLXI (situé sur le chromosome 10q34) et TLX3 (situé sur le chromosome 5 q35) sont dérégulés consécutivement à des translocations chromosomiques et de manière mutuellement exclusive dans environ 30 à $35 \%$ des LAL-T adultes et pédiatriques. Dans ces affections, les protéines dérivées, TLXI et TLX3, se trouvent alors exprimées de façon ectopique par les cellules tumorales. Ces LAL-T (appelées ci-après $T L X^{+}$) se caractérisent par un stade spécifique de blocage de la différenciation thymique dénommé «cortical précoce». Ce stade se définit notamment par la présence de réarrangements $V(D) J d u$ gène $T C R \beta$ sans expression détectable d'un récepteur membranaire TCR $\alpha \beta$ [6], suggérant une absence de recombinaison $V(D)$ J au locus TCR $\alpha$ qui serait spécifique de ces formes de leucémies. Dans les thymocytes immatures en voie de développement, les étapes de la mise en place ordonnée des réarrangements $\mathrm{V}(\mathrm{D}) \mathrm{J}$ aux locus $T C R \gamma, \delta, \beta$ et $\alpha$ sont reconnues comme étant critiques pour la différenciation des cellules Tgd et Tab [7]. Ainsi, les recombinaisons aux locus TCR $\gamma, \delta$ et $\beta$ se produisent dans les thymocytes immatures précoces $\operatorname{CD} 4^{-} \operatorname{CD} 8^{-}$(double négatifs), alors que celles impliquant les segments de gène Va et Ja du locus TCR $\alpha$ (qui provoquent la délétion du locus intermédiaire $T C R \delta$ ) sont plus tardives, se produisant dans les thymocytes $\mathrm{CD}^{+} \mathrm{CD}^{+}$(double positifs). L'activation de la région génomique contenant les segments Ja pour les recombinaisons Va-Ja est placée sous le contrôle d'un élément de régulation appelé enhancer $\alpha(\varepsilon \alpha)$. Cet élément comporte une région centrale (core) qui comprend des sites de fixation pour les facteurs de transcription LEFl, RUNXI (runt-related transcription factor 1 ) et ETS1 ( $v$-ets erythroblastosis virus E26 oncogene homolog 1), facteurs dont le recrutement s'avère essentiel pour son activité [8].

\section{Mécanisme moléculaire \\ de la répression \\ de l'enhancer $\alpha(\varepsilon \alpha)$}

Dans un travail récent présenté dans la revue Cancer Cell [9], nos équipes de recherche à l'Hôpital Necker-Enfants malades et au Centre d'immunologie de Marseille-Luminy ont d'abord confirmé que les LAL-T TLX+ ${ }^{+}$présentent bien un défaut de recombinaison de la partie $\int \alpha$ du locus $T C R \alpha$. Nous avons ensuite établi l'existence d'un état de répression épigénétique spécifique au locus $T C R-J \alpha$, caractérisé notamment par la présence de marques d'inactivation génique du type triméthylation des résidus lysine 27 au niveau des régions amino-terminales de l'his- 


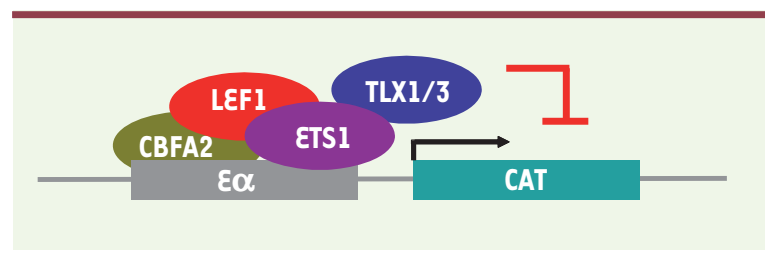

Figure 1. Mécanisme moléculaire de la répression de $\varepsilon \alpha$. La cotransfection dans des cellules HeLa de la région core $\varepsilon \alpha$ associée au gène rapporteur CAT et des facteurs de transcription AMLI, ETSl et LEFl nécessaires à son activité de stimulation de la transcription, permet de mettre en évidence la répression transcriptionnelle induite en présence des oncoprotéines TLXI ou TLX3.

tone $\mathrm{H} 3$ des nucléosomes (marques H3K27me3). Il est probable que la conformation chromatinienne associée à ce marquage répressif interdise l'accessibilité de cette région du génome à la machinerie de recombinaison V(D)J (la recombinase RAGl/ RAG2 y définit normalement ce qu'il est convenu d'appeler un recombination center [10]), inhibant ainsi la formation de réarrangements $V \alpha-J \alpha$. En conséquence, la poursuite de la différenciation cellulaire $T$ normale vers le stade TCR $\alpha \beta^{+}$est bloquée. Un système de transfection utilisant le gène rapporteur $\varepsilon \alpha$-CAT (chloramphenicol acetyltransferase), reconstituant un élément $\varepsilon \alpha$ core fonctionnel dans des cellules HeLa (Figure 1), a ensuite permis la mise en évidence, lorsque des protéines $T L X I / 3$ étaient cotransfectées, d'une forte répression de l'activité transcriptionnelle dépendante de $\varepsilon \alpha$.

Enfin, nous avons également levé un coin du voile sur les mécanismes moléculaires à l'origine de ces blocages en démontrant que les oncoprotéines TLXI et TLX3, par l'intermédiaire d'une interaction protéine-protéine impliquant I'homéodomaine, lient le facteur de transcription ETS I I via son domaine DBD (DNA binding domain) (Figures 1 et 2). Cette interaction a pour effet le recrutement des oncoprotéines TLX au niveau de $\varepsilon \alpha$, le marquage épigénétique répressif (H3K27me3) évoqué plus haut, l'inactivation des réarrangements $V \alpha$-J $\alpha$ et l'arrêt du processus de maturation. Le blocage de différenciation sans apoptose qui en résulte expose la cellule à d'autres événements oncogéniques dont l'accumulation au cours de l'histoire naturelle de la maladie se traduit in fine par l'émergence du clone leucémique.

\section{La restauration de la différenciation} des cellules leucémiques $\mathrm{TLX}^{+}$entraîne leur apoptose :

une piste thérapeutique?

De manière très remarquable, l'inactivation directe (knock-down de l'expression de TLX via des shARN délivrés via un vecteur lentiviral) ou indirecte (surexpression lentivirale d'un récepteur TCR $\alpha \beta$ exogène) de ce processus répressif a pour conséquence une reprise de la différenciation T suivie d'une mort cellulaire par apoptose. Dans ce système, des cellules SIL-ALL $\left(T L X I^{+}\right)$ont été transfectées à l'aide d'un lentivirus tricistronique induisant in fine l'expression d'une protéine GFP (green fluorescent protein)-TCR $\alpha \beta$. Les cellules

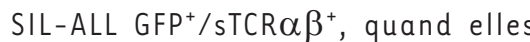
sont cultivées sur un stroma cellulaire OP9-DLl ${ }^{l}$ en présence d'un cocktail de cytokines (IL [interleukine]-7, FLT3-L et SCF [stem cell factor]), subissent une apoptose massive en comparaison des cellules contrôles $\mathrm{GFP}^{+} / \mathrm{sTCR} \alpha \beta^{-}$. De façon notable, ce phénomène d'apoptose induite n'est pas observé dans les conditions de culture standard en l'absence de stroma cellulaire OP9-DL1. Les

${ }^{1}$ II s'agit de cellules stromales murines OP9 surexpriman delta 1 , un des ligands des récepteurs Notch. L'interaction Notch-Delta est indispensable à la différenciation des thymocytes.

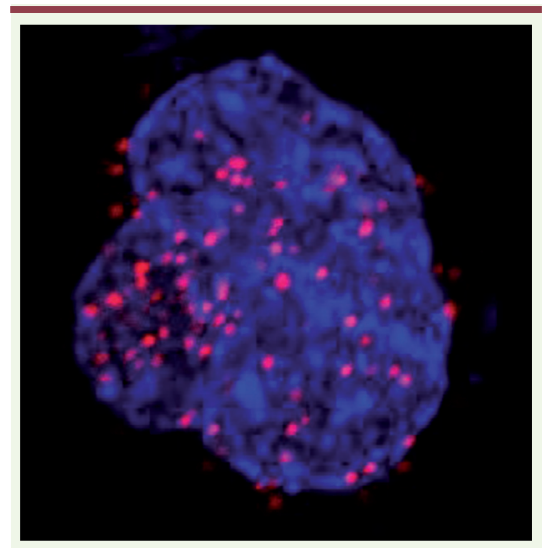

Figure 2. Mise en évidence de l'interaction TLXETS1. Vue microscopique d'un noyau de cellule $\mathrm{TLXI}^{+}$après marquages intranucléaires à l'aide d'anticorps primaires anti-TLXI et antiETS1, révélés par l'intermédiaire d'anticorps secondaires et test de fluorescence PLA (pour proximity ligation assay). Dans ce type de test, les anticorps secondaires sont couplés à des fluorochromes ainsi qu'à des oligonucléotides de séquences complémentaires. Des signaux fluorescents (spots rouges) sont générés si ces derniers anticorps peuvent se lier par hybridation oligonucléotidique, ce qui en pratique traduit l'existence de complexes protéiques au sein desquels les protéines cibles (ici TLXI et ETSl) sont étroitement associées (distantes de moins de $40 \mathrm{~nm}$ ).

cellules restent ainsi dépendantes du blocage de maturation dont la levée semble incompatible avec leur survie, et ce malgré l'accumulation de nombreux autres évènements oncogéniques habituellement présents dans ces hémopathies. II n'est pas inconcevable non plus qu'un signal TCR fort dans une cellule bloquée au stade de la $\beta$-sélection soit interprété comme un signal de sélection négative thymique et provoque l'apoptose. Ainsi, l'accumulation des lésions oncogénétiques n'est pas suffisante à elle seule pour assurer la survie cellulaire des blastes leucémiques dans ce modèle de LAL-T. Le blocage de différenciation que crée l'effet «TCR manquant » est indispensable à la survie des cellules leucémiques $\operatorname{TLX}^{+}$. Ces résultats ouvrent 
des perspectives originales de thérapie ciblée différenciante dans un modèle original d'hémopathie lymphoïde. $\diamond$ Deciphering functional activity of TLX homeodomain oncogenes in T-ALL: a clue for a differentiating therapy?

\section{LIENS D'INTÉRÊT}

Les auteurs déclarent n'avoir aucun lien d'intérêt concernant les données publiées dans cet article.

\section{RéFÉRENCES}

1. Asnafi V, Beldjord K, Boulanger $\varepsilon$, et al. Analysis of TCR, PT alpha, and RAG-1 in T-acute lymphoblastic leukemias improves understanding of early human T-lymphoid lineage commitment. Blood $2003 ; 101$ : 2693-703.
2. Ferrando A, Neuberg D, Dodge RK, et al. Adult T-cell ALL patients whose lymphoblasts express the HOXIl oncogene have an excellent prognosis when treated with chemotherapy and are not candidates for allogeneic bone marrow transplantaton in first remission. Blood 2004 ; 100 : 154a.

3. Pui CH, Robison LL, Look AT. Acute lymphoblastic leukaemia. Lancet 2008 ; 371 : 1030-43.

4. Aifantis I, Raetz $\varepsilon$, Buonamici S. Molecular pathogenesis of T-cell leukaemia and lymphoma. Nat Rev Immunol 2008 ; 8 : 380-90.

5. Argiropoulos B, Humphries RK. Hox genes in hematopoiesis and leukemogenesis. Oncogene 2007 ; 26 : 6766-76.

6. Asnafi V, Beldjord K, Libura M, et al. Age-related phenotypic and oncogenic differences in T-cell acute lymphoblastic leukemias may reflect thymic atrophy. Blood 2004 ; 104 : 4173-80.

7. Dik WA, Pike-Overzet K, Weerkamp F, et al. New insights on human $T$ cell development by quantitative $T$ cell receptor gene rearrangement studies and gene expression profiling. J Exp Med $2005 ; 201$ : 1715-23.

\section{NOUVELLE}

\section{L'évolution darwinienne d'un système génétique non conventionnel met en évidence un ancêtre potentiel de I'ARN}

Annabelle Gillig
> La question de l'apparition de la vie sur Terre est l'une des plus fascinantes dans le domaine scientifique. L'hypothèse d'un monde d'acides ribonucléiques (ARN) est fondée sur le principe que la vie sur Terre est apparue au cours des temps géologiques au travers de I'ARN, capable de catalyser des réactions chimiques et d'être le support d'une information génétique [1]. II n'est cependant pas évident que I'ARN fût le matériel génétique initial. En effet, les difficultés liées à la synthèse prébiotique du ribose, composant de I'ARN, et à la réplication non enzymatique de ce dernier, ont suscité un vif intérêt pour la théorie d'un monde pré-ARN. Cette théorie considère que I'ARN a été précédé dans l'évolution de la vie par un matériel génétique plus simple et plus accessible dans les temps primitifs $[2,3]$.
Afin de comprendre l'origine et l'évolution de la vie, il est fondamental de déterminer les conditions nécessaires à l'apparition d'un monde d'ARN. Dans ce but, une étude systématique a été réalisée sur des systèmes d'acides nucléiques potentiellement naturels afin de déterminer leur capacité à former des appariements de type Watson-Crick et donc à transmettre l'information génétique [4]. Un métabolisme primitif nécessite également que ces polymères soient capables de se replier dans des structures tertiaires stables ayant des fonctionnalités élaborées telles que la reconnaissance d'un ligand et la catalyse [5].

Parmi les systèmes étudiés, l'acide nucléique (3', 2')- $\alpha$-L-thréose (ANT, Figure 1) pourrait jouer le rôle de précurseur de l'ARN en raison de sa simplicité structurale - son squelette est constitué de thréoses qui ont un carbone de moins
8. Giese K, Kingsley C, Kirshner JR, Grosschedl R. Assembly and function of a TCR alpha enhancer complex is dependent on LEF-1-induced DNA bending and multiple protein-protein interactions. Genes Dev $1995 ; 9$ : 995-1008.

9. Dadi S, Le Noir S, Payet-Bornet D, et al. TLX Homeodomain oncogenes mediate T cell maturation arrest in T-ALL via interaction with ETSl and suppression of TCR $\alpha$ gene expression. Cancer Cell $2012 ; 21: 563-76$.

10. Ji $Y$, Resch W, Corbett, $\varepsilon$, et al. The in vivo pattern of binding of RAG1 and RAG2 to antigen receptor loci. Cell $2010 ; 141: 419-31$.
Biodesign Institute, Arizona State University, 1001 S. McAllister Ave., Tempe, AZ 85287, États-Unis.

annabelle.gillig@asu.edu

que les riboses de l'ARN - et de sa capacité à former des structures hélicoïdales stables avec des brins complémentaires d'ANT ou d'ARN [6-8].

La sélection in vitro, ou évolution moléculaire, est une méthode de choix dans l'étude des propriétés fonctionnelles des acides nucléiques. Cette approche a cependant toujours été limitée à I'ADN, à I'ARN ou à d'autres analogues structurels puisque ces derniers sont les seuls qui disposent d'enzymes connues capables de réaliser la transcription, la transcription inverse et l'amplification de l'information génétique. Appliquer cette méthode à un système génétique artificiel tel que l'ANT nécessite :

- des polymérases capables de transcrire une librairie de séquences aléatoires d'ADN en ANT;

- une méthode de sélection qui permet d'isoler les individus présentant une 